\title{
SOME EXAMPLES OF BANDS WITH TWO COMPONENTS
}

\author{
Ferran Cedó And Elena Rodríguez-Jorge
}

\begin{abstract}
A band is a semigroup whose elements are idempotents. The paper is motivated by some open problems concerning linear representations of bands. A counterexample to a conjecture stated in [1] is given. On the other hand, it is proved that there exist linear bands with two components such that the associated commutative $K$-algebra constructed in $[\mathbf{1}]$ cannot be embedded into any Noetherian ring, even in the case where this algebra is reduced.
\end{abstract}

\section{Introduction}

Recall that a band is a semigroup $S$ whose elements are idempotents, that is, $e^{2}=e$ for all $e \in S$. The problem of embeddability of a band into the multiplicative semigroup of matrices over a field was first raised in [4], and some advances in its study can be found in recent papers ([1], $[2],[3])$.

Recall that a rectangular band is a band $E$ such that $x y z=x z$ for all $x, y, z \in E$. Equivalently, $E \cong \mathcal{M}(\{1\}, X, Y ; P)$, a completely simple semigroup over the trivial group and with a sandwich matrix $P=\left(p_{y x}\right)$ where $p_{y x}=1$ for all $x \in X, y \in Y$. It is known that for any band $S$ there exists a semilattice (a commutative band) $\Gamma$ such that $S=\bigcup_{\gamma \in \Gamma} S_{\gamma}$, where $S_{\gamma}$ are disjoint rectangular subbands of $S$ such that $S_{\gamma} S_{\delta} \subseteq S_{\gamma \delta}$, for all $\gamma, \delta \in \Gamma$. Here $S_{\gamma}$ are called the components of $S$. For more details we refer to $[\mathbf{6}]$.

It is well-known that every linear band has finitely many components and it is triangularizable, see $[\mathbf{5}, \S 4.4]$ or $[\mathbf{7}]$. It is also known that for any field $K$ and any rectangular band $F$, the semigroup algebra $K[F]$

2000 Mathematics Subject Classification. Primary: 20M25, 16S36, 16R20.

Key words. Band, semigroup algebra, triangular matrices.

Research partially supported by grants of MICIN-FEDER (Spain) MTM2008-06201C02-01, Generalitat de Catalunya 2009 SGR 1389. 
embeds into $M_{3}(L)$, for some field extension $L$ of $K$, see [1] or [4]. However, there are examples of non linear bands with two components (see $[\mathbf{1}],[\mathbf{2}]$ and $[\mathbf{4}]$ ).

Let $S$ be a semigroup. We denote by $S^{1}$ the monoid $S \cup\{1\}$, where 1 is the identity element (if $S$ is a monoid then we assume that $S=S^{1}$ ). Let $s, t \in S^{1}$. The right annihilator $\mathrm{r}$. $\operatorname{ann}_{S}(s-t)=\{x \in S \mid s x=t x\}$ is a right ideal of $S$, and similarly l. $\operatorname{ann}_{S}(s-t)$, the left annihilator in $S$, is a left ideal of $S$. The right annihilator $\mathrm{r} . \operatorname{ann}_{S}(s)=\{(x, y) \in S \times S \mid$ $s x=s y\}$ is a right congruence on $S$, and the left annihilator $1 . \operatorname{ann}_{S}(s)$ is a left congruence on $S$. In [1] it is conjectured that if $S$ is a band with finitely many components such that every chain of one-sided ideals of the form r. $\operatorname{ann}_{S}(s-t), \mathrm{l} \cdot \mathrm{ann}_{S}(s-t), s, t \in S^{1}$, is of bounded length and every chain of one-sided congruences of the form r. $\operatorname{ann}_{S}(s), l \cdot \operatorname{ann}_{S}(s)$, $s \in S$, has bounded length, then $S$ embeds into the ring of matrices of some size over a field $L$. In Section 2 , we give a counterexample to this conjecture and propose a new one (Conjecture 2.3) by strengthening the conditions on annihilators and congruences. These new conditions are necessary for the band to be linear, but it is unknown whether they are sufficient or not.

In [1] , it is also proven that, for any field $K$ and any band $S$ with finitely many components, the semigroup algebra $K[S]$ embeds into $T_{n}(A)$ for a commutative $K$-algebra $A$, where $T_{n}(A)$ denotes the algebra of upper triangular matrices $m=\left(m_{i j}\right)$ over $A$ with $m_{i i} \in K$ for every $i=1, \ldots, n$. In the particular case of bands with two components, a commutative $K$-algebra $\bar{R}$ is constructed, together with an explicit embedding of $K[S]$ into $T_{7}(\bar{R})$.

In [2], it is proven that, if the band $S$ with components $E$ and $F$ satisfies the condition $E F E=F$, then the associated $K$-algebra $\bar{R}$ is reduced. By Goldie's Theorem, the latter property is a necessary condition for $\bar{R}$ to embed into a finite product of fields, in which case we could embed $T_{7}(\bar{R})$, and therefore $S$, into matrices over some field. In this case a sufficient condition for $S$ to be linear would be that $\bar{R}$ satisfies ascending chain condition on annihilators. Thus, a natural question is whether $S$ satisfying ascending chain condition on annihilators and congruences implies $\bar{R}$ to satisfy ascending chain condition on annihilators. One could expect that the linearity of $S$ could be equivalent to the linearity of $\bar{R}$. But this is not the case; in Section 3, we give an example of a linear band with two components $E$ and $F$ satisfying $E F E=F$ such that its associated commutative $K$-algebra $\bar{R}$ has an infinite chain of annihilator ideals. 


\section{Counterexample}

Let $S$ be a band with two components $E$ and $F$ such that $E F, F E \subseteq$ $F$. We may assume without loss of generality that there exist non-empty sets $I, J, A, B$ such that $E=\left\{e_{a, b} \mid a \in A, b \in B\right\}$ and $F=\left\{f_{i, j} \mid i \in\right.$ $I, j \in J\}$, and maps $\alpha: A \times B \times I \rightarrow I$ and $\beta: J \times A \times B \rightarrow J$ such that the product in $S$ is given by the rules:

- $e_{a, b} e_{c, d}=e_{a, d}$ for all $a, c \in A$ and for all $b, d \in B$,

- $f_{i, j} f_{k, l}=f_{i, l}$ for all $i, k \in I$ and for all $j, l \in J$,

- $e_{a, b} f_{i, j}=f_{\alpha(a, b, i), j}$ for all $a \in A, b \in B, i \in I$ and $j \in J$,

- $f_{i, j} e_{a, b}=f_{i, \beta(j, a, b)}$ for all $a \in A, b \in B, i \in I$ and $j \in J$

and, for all $a, c \in A, b, d \in B, i \in I$ and $j \in J, \alpha(a, b, \alpha(c, d, i))=$ $\alpha(a, d, i)$ and $\beta(\beta(j, a, b), c, d)=\beta(j, a, d)$.

A band $S$ is said to be linear if there exists a monomorphism from $S$ to the multiplicative semigroup $M_{n}(K)$ for some positive integer $n$ and some field $K$. We want to know when a band with two components is linear. Linearity of a band implies certain finiteness conditions on its annihilators. Let $L$ be a field. If $S \subseteq M_{n}(L)$ is a linear semigroup then every chain of annihilator (one-sided) ideals of the algebra $\operatorname{lin}_{L}(S) \subseteq M_{n}(L)$ has length bounded by $n$. This motivated the following conjecture appeared in $[\mathbf{1}]$.

Conjecture 2.1. Let $S$ be a band with finitely many components. Assume that every chain of one-sided ideals of the form r. $\operatorname{ann}_{S}(s-$ $t)$, l. $\operatorname{ann}_{S}(s-t)$, with $s, t \in S^{1}$, is of bounded length and every chain of one-sided congruences of the form r. $\operatorname{ann}_{S}(s), 1 . \operatorname{ann}_{S}(s)$, with $s \in S$, has bounded length. Then, $S$ (or maybe also $K[S]$ for any field $K$ ) embeds into matrices over some field.

Theorem 2.2. There exists a counterexample to Conjecture 2.1.

Proof: Let $E=\left\{e_{n} \mid n \in \mathbb{N}\right\}, F=\left\{f_{(m, k)} \mid m \in \mathbb{N}, k \in\{0,1\}\right\}$ and $S=E \cup F$. Define in $S$ the following product:

- $e \cdot e^{\prime}=e$ for all $e, e^{\prime} \in E$,

- $f \cdot s=f$ for all $f \in F$ and for all $s \in S$,

- $e_{n} \cdot f_{(m, k)}=f_{\left(m, \delta_{m, n}\right)}$, where $\delta_{m, n}$ denotes the Kronecker's delta, for all $n, m \in \mathbb{N}$ and for all $k \in\{0,1\}$.

Then, we will see that $S$ is a non-linear band with components $E$ and $F$ that satisfies the chain conditions stated in Conjecture 2.1. 
First of all, we need to see that $S$ is a band. If we define

$$
\alpha: \mathbb{N} \times(\mathbb{N} \times\{0,1\}) \rightarrow(\mathbb{N} \times\{0,1\})
$$

by $\alpha(n,(m, k))=\left(m, \delta_{n, m}\right)$, then, in order to check the associativity of the product in $S$, it is enough to see that $\alpha\left(n, \alpha\left(n^{\prime},(m, k)\right)\right)=$ $\alpha(n,(m, k))$ for all $n, n^{\prime}, m \in \mathbb{N}$ and for all $k \in\{0,1\}$.

Let $n, n^{\prime}, m \in \mathbb{N}$ and $k \in\{0,1\}$. Then

$$
\alpha\left(n, \alpha\left(n^{\prime},(m, k)\right)\right)=\alpha\left(n,\left(m, \delta_{n^{\prime}, m}\right)\right)=\left(m, \delta_{n, m}\right)=\alpha(n,(m, k)) .
$$

Now, it is clear that $S$ is a band with components $E$ and $F$.

Suppose that $S$ is linear. Thus, there exist a positive integer $n$, a field $K$ and an embedding $\varphi: S \rightarrow M_{n}(K)$. Let $\operatorname{lin}_{K}(S)$ denote the subalgebra of $M_{n}(K)$ generated by $\varphi(S)$. Consider the following left annihilators in $\operatorname{lin}_{K}(S)$ :

$$
J_{p}=\left\{x \in \operatorname{lin}_{K}(S) \mid x \varphi\left(f_{(m, 0)}\right)=0 \text { for all } m \leq p\right\},
$$

for $p \in \mathbb{N}$. Clearly, $J_{1} \supseteq J_{2} \supseteq \cdots \supseteq J_{p} \supseteq \cdots$.

Since $\operatorname{lin}_{K}(S)$ is a subalgebra of $M_{n}(K)$, it satisfies the descending chain condition on annihilators. Hence, there exists $p \in \mathbb{N}$ such that $J_{q}=J_{p}$ for all $q \geq p$. Note that, for all $m \leq p$,

$$
\begin{aligned}
\left(\varphi\left(e_{p+1}\right)-\varphi\left(e_{p+2}\right)\right) \varphi\left(f_{(m, 0)}\right) & =\varphi\left(e_{p+1}\right) \varphi\left(f_{(m, 0)}\right)-\varphi\left(e_{p+2}\right) \varphi\left(f_{(m, 0)}\right) \\
& =\varphi\left(e_{p+1} f_{(m, 0)}\right)-\varphi\left(e_{p+2} f_{(m, 0)}\right) \\
& =\varphi\left(f_{(m, 0)}\right)-\varphi\left(f_{(m, 0)}\right) \\
& =0,
\end{aligned}
$$

and

$$
\begin{aligned}
\left(\varphi\left(e_{p+1}\right)-\varphi\left(e_{p+2}\right)\right) \varphi\left(f_{(p+1,0)}\right) & =\varphi\left(e_{p+1}\right) \varphi\left(f_{(p+1,0)}\right)-\varphi\left(e_{p+2}\right) \varphi\left(f_{(p+1,0)}\right) \\
& =\varphi\left(e_{p+1} f_{(p+1,0)}\right)-\varphi\left(e_{p+2} f_{(p+1,0)}\right) \\
& =\varphi\left(f_{(p+1,1)}\right)-\varphi\left(f_{(p+1,0)}\right) \\
& \neq 0,
\end{aligned}
$$

since $\varphi$ is inyective. Hence $\varphi\left(e_{p+1}\right)-\varphi\left(e_{p+2}\right) \in J_{p} \backslash J_{p+1}$, a contradiction. 
Therefore, $S$ is not linear.

We now calculate all the different annihilators of the forms r. $\operatorname{ann}_{S}(s)$, l. $\operatorname{ann}_{S}(s)$, r. $\operatorname{ann}_{S}(s-r)$ and l. $\operatorname{ann}_{S}(s-r)$.

1. $\operatorname{ann}_{S}\left(e_{n}\right)=\{(s, s) \mid s \in S\}$,

r. $\operatorname{ann}_{S}\left(e_{n}\right)=\left\{\left(f_{\left(m, k_{1}\right)}, f_{\left(m, k_{2}\right)}\right) \mid m \in \mathbb{N}, k_{1}, k_{2} \in\{0,1\}\right\} \cup E \times E$,

1. $\operatorname{ann}_{S}\left(f_{(m, k)}\right)=\{(s, s) \mid s \in S\} \cup\left\{\left(e_{n}, e_{n^{\prime}}\right) \mid n \neq m\right.$ and $\left.n^{\prime} \neq m\right\}$ $\cup\left\{\left(e_{n}, f_{(m, 0)}\right),\left(f_{(m, 0)}, e_{n}\right) \mid n \neq m\right\}$

r. $\operatorname{ann}_{S}\left(f_{(m, k)}\right)=S \times S$, $\cup\left\{\left(e_{m}, f_{(m, 1)}\right),\left(f_{(m, 1)}, e_{m}\right)\right\}$,

1. $\operatorname{ann}_{S}\left(1-e_{n}\right)=S$,

r. $\operatorname{ann}_{S}\left(1-e_{n}\right)=\left\{e_{n}\right\} \cup\left\{f_{(m, 0)} \mid n \neq m\right\} \cup\left\{f_{(n, 1)}\right\}$,

1. $\operatorname{ann}_{S}\left(1-f_{(m, k)}\right)=F$,

r. $\operatorname{ann}_{S}\left(1-f_{(m, k)}\right)=\left\{f_{(m, k)}\right\}$,

1. $\operatorname{ann}_{S}\left(f_{(m, k)}-f_{\left(m^{\prime}, k^{\prime}\right)}\right)= \begin{cases}F & \text { if } m \neq m^{\prime}, \\ S & \text { if } m=m^{\prime}\end{cases}$

r. $\operatorname{ann}_{S}\left(f_{(m, k)}-f_{\left(m^{\prime}, k^{\prime}\right)}\right)= \begin{cases}\emptyset & \text { if }(m, k) \neq\left(m^{\prime}, k^{\prime}\right), \\ S & \text { if }(m, k)=\left(m^{\prime}, k^{\prime}\right),\end{cases}$

1. $\operatorname{ann}_{S}\left(e_{n}-f_{(m, k)}\right)=F$,

r. $\operatorname{ann}_{S}\left(e_{n}-f_{(m, k)}\right)= \begin{cases}\left\{f_{(m, 0)}, f_{(m, 1)}\right\} & \text { if } n \neq m \text { and } k=0, \\ \emptyset & \text { if } n=m \text { and } k=0, \\ \emptyset & \text { if } n \neq m \text { and } k=1, \\ \left\{f_{(m, 0)}, f_{(m, 1)}\right\} & \text { if } n=m \text { and } k=1,\end{cases}$

1. $\operatorname{ann}_{S}\left(e_{n}-e_{n^{\prime}}\right)=S$,

r. $\operatorname{ann}_{S}\left(e_{n}-e_{n^{\prime}}\right)= \begin{cases}\left\{f_{(m, k)} \mid k \in\{0,1\}, n \neq m \text { and } n^{\prime} \neq m\right\} & \text { if } n \neq n^{\prime} \\ S & \text { if } n=n^{\prime}\end{cases}$

Clearly the length of any chain of such annihilators is bounded by 5 , so $S$ satisfies the chain conditions stated in Conjecture 2.1.

Therefore $S$ is a counterexample to Conjecture 2.1 .

Now we know that an existence of a bound on the lengths of chains of annihilators of elements is not sufficient for linearity of a band. However, in view of the above example one might propose a new conjecture. 
Conjecture 2.3. Let $S$ be a band with finitely many components. Assume that every chain of one-sided ideals of the form r. $\operatorname{ann}_{S}\left(s_{1}-\right.$ $\left.t_{1}, \ldots, s_{n}-t_{n}\right)$, l. $\operatorname{ann}_{S}\left(s_{1}-t_{1}, \ldots, s_{n}-t_{n}\right)$, with $s_{i}, t_{i} \in S^{1}$ for all $n \in \mathbb{N}$ and for all $1 \leq i \leq n$, is of bounded length and every chain of onesided congruences of the form r. $\operatorname{ann}_{S}\left(s_{1}, \ldots, s_{n}\right), l_{\cdot} \operatorname{ann}_{S}\left(s_{1}, \ldots, s_{n}\right)$, with $s_{i} \in S$ for all $n \in \mathbb{N}$ and for all $1 \leq i \leq n$, has bounded length. Then, $S$ (or maybe also $K[S]$ for any field $K$ ) embeds into matrices over some field.

This new conjecture remains unsolved, even in the simplest (nontrivial) case, namely, the case of bands with two components. Since if $S \subseteq M_{n}(L)$ then every chain of annihilator (one-sided) ideals of the algebra $\operatorname{lin}_{L}(S) \subseteq M_{n}(L)$ has length bounded by $n$, it is clear that the conditions of Conjecture 2.3 are necessary for $S$ to be linear.

\section{The commutative $K$-algebra associated to bands with two components}

Let $S$ be a band with two components, $E$ and $F$, such that $F$ is an ideal of $S$. We will use the same notation as in Section 2. Then, we may assume that $E=\left\{e_{a, b} \mid a \in A, b \in B\right\}, F=\left\{f_{i, j} \mid i \in I, j \in J\right\}$ and that the product in $S$ is given by:

- $e_{a, b} e_{c, d}=e_{a, d}$ for all $a, c \in A$ and for all $b, d \in B$,

- $f_{i, j} f_{k, l}=f_{i, l}$ for all $i, k \in I$ and for all $j, l \in J$,

- $e_{a, b} f_{i, j}=f_{\alpha(a, b, i), j}$ for all $a \in A, b \in B, i \in I$ and $j \in J$,

- $f_{i, j} e_{a, b}=f_{i, \beta(j, a, b)}$ for all $a \in A, b \in B, i \in I$ and $j \in J$,

for some maps $\alpha: A \times B \times I \rightarrow I$ and $\beta: J \times A \times B \rightarrow J$ satisfying

$$
\begin{aligned}
& \alpha(a, b, \alpha(c, d, i))=\alpha(a, d, i), \\
& \beta(\beta(j, a, b), c, d)=\beta(j, a, d),
\end{aligned}
$$

for all $a, c \in A, b, d \in B, i \in I$ and $j \in J$.

Let $K$ be a field. Recall the construction in [1] of a commutative $K$-algebra $\bar{R}$ associated to a band $S$ with two components such that $S$ embeds into $T_{7}(\bar{R})$.

Let $X=\left\{x_{i} \mid i \in I\right\}, Y=\left\{y_{j} \mid j \in J\right\}, Z=\left\{z_{a} \mid a \in A\right\}, Z^{\prime}=\left\{z_{a}^{\prime} \mid\right.$ $a \in A\}, T=\left\{t_{b} \mid b \in B\right\}$ and $T^{\prime}=\left\{t_{b}^{\prime} \mid b \in B\right\}$ be pairwise disjoint sets of commuting indeterminates over $K$. Let $R=K\left[X \cup Y \cup Z \cup Z^{\prime} \cup T \cup T^{\prime}\right]$ be the polynomial ring on these indeterminates with coefficients in $K$. Let $M$ be the ideal of $R$ generated by 
(a) $t_{b} x_{i}-t_{d} x_{k}$ for all $i, k \in I$ and all $b, d \in B$ such that there exists $a \in A$ satisfying $\alpha(a, b, i)=\alpha(a, d, k)$,

(b) $z_{a} t_{b} x_{i}-z_{c} t_{d} x_{k}$ for all $i, k \in I$, all $a, c \in A$ and all $b, d \in B$ such that $\alpha(a, b, i)=\alpha(c, d, k)$

(c) $y_{j} z_{a}^{\prime}-y_{l} z_{c}^{\prime}$ for all $j, l \in J$ and all $a, c \in A$ such that there exists $b \in B$ satisfying $\beta(j, a, b)=\beta(l, c, b)$,

(d) $y_{j} z_{a}^{\prime} t_{b}^{\prime}-y_{l} z_{c}^{\prime} t_{d}^{\prime}$ for all $j, l \in J$, all $a, c \in A$ and all $b, d \in B$ such that $\beta(j, a, b)=\beta(l, c, d)$.

Then $\bar{R}=R / M$. We call $\bar{R}$ the commutative $K$-algebra associated to $S$.

Due to an intimate relation between the definition of $\bar{R}$ and the structure of $S$, one could expect that the linearity of $S$ implies the linearity of $\bar{R}$. In $[\mathbf{2}]$ we began this investigation and we proved that if the components $E$ and $F$ of $S$ satisfy $E F E=F$, then $\bar{R}$ is reduced. Note that, by Goldie's Theorem, a commutative reduced ring embeds into a finite product of fields if and only if it satisfies the ascending chain condition on annihilators. Thus, one might expect that the chain conditions on annihilators in $S$ could imply the chain condition on annihilators in $\bar{R}$. Note that this would give a way to prove Conjecture 2.3 in the case of bands of two components $E$ and $F$ such that $E F E=F$. However, in Theorem 3.1 we will prove that there exists a linear band with two components $E$ and $F$ satisfying $E F E=F$ such that $\bar{R}$ cannot be embedded into any Noetherian ring.

Theorem 3.1. For any field $K$ there exists a linear band with two components $E$ and $F$ satisfying $E F E=F$ such that the commutative $K$-algebra $\bar{R}$ associated to it cannot be embedded into any Noetherian ring.

Proof: Let $I=\mathbb{N} \times \mathbb{N}$ and $A=\mathbb{N} \cup\{\infty\}$. Let $E=\left\{e_{a} \mid a \in A\right\}$ and $F=\left\{f_{i} \mid i \in I\right\}$. We define the following product on $S=E \cup F$ :

- $e e^{\prime}=e$ for all $e \in E$,

- $f s=f$ for all $f \in F, s \in S$,

- $e_{a} f_{i}=f_{\alpha(a, i)}$, where $\alpha: A \times I \rightarrow I$ is defined by $\alpha\left(a,\left(i_{1}, i_{2}\right)\right)=$ $\left(\alpha_{i_{2}}(a), i_{2}\right)$ for all $a \in \mathbb{N} \cup\{\infty\}$, with

$$
\alpha_{i_{2}}(a)=\left\{\begin{array}{ll}
a & \text { if } a \notin\left\{i_{2}+1, \infty\right\} \\
i_{2} & \text { if } a=i_{2}+1 \\
i_{2}+1 & \text { if } a=\infty
\end{array} .\right.
$$

Clearly, with this product, every element in $S$ is idempotent. We check that the product is associative. Note that, for $a, c \in A, i=\left(i_{1}, i_{2}\right) \in I$, 
we have that $\alpha(a, \alpha(c, i))=\alpha\left(a,\left(\alpha_{i_{2}}(c), i_{2}\right)\right)=\left(\alpha_{i_{2}}(a), i_{2}\right)=\alpha(a, i)$. It is now easy to see that

- $e(f s)=e f=(e f) s$ for all $e \in E, f \in F$ and $s \in S$,

- $f\left(s_{1} s_{2}\right)=f=\left(f s_{1}\right) s_{2}$ for all $f \in F, s_{1}, s_{2} \in S$,

- $e_{1}\left(e_{2} e_{3}\right)=e_{1}=\left(e_{1} e_{2}\right) e_{3}$ for all $e_{1}, e_{2}, e_{3} \in E$,

- $e_{a}\left(e_{c} f_{i}\right)=f_{\alpha(a, \alpha(c, i))}=f_{\alpha(a, i)}=\left(e_{a} e_{c}\right) f_{i}$ for all $a, c \in A, i \in I$.

Therefore, $S$ is a band, and by definition, it is clear that $E$ and $F$ are the only two components of $S$. Note that $E F E=F$, since, for all $f_{\left(i_{1}, i_{2}\right)} \in$ $F$, if $i_{1} \neq i_{2}+1$, there exists $e_{i_{1}} \in E$ such that $e_{i_{1}} f_{\left(i_{1}, i_{2}\right)} e_{i_{1}}=f_{\left(i_{1}, i_{2}\right)}$ and $e_{\infty} f_{\left(i_{2}+1, i_{2}\right)} e_{\infty}=f_{\left(i_{2}+1, i_{2}\right)}$.

Let us define an equivalence relation in $I$ by $i \sim k$ if and only if $\alpha(a, i)=\alpha(a, k)$ for all $a \in A$. Equivalently, $i \sim k$ if and only if there exists $a \in A$ such that $\alpha(a, i)=\alpha(a, k)$.

Claim. For $a, c \in A$ and $i, k \in I, \alpha(a, i)=\alpha(c, k)$ if and only if $i \sim k$ and $\alpha(a, i)=\alpha(c, i)$.

In order to prove the claim, assume that $\alpha(a, i)=\alpha(c, k)$. Then, $\alpha(a, k)=\alpha(a, \alpha(c, k))=\alpha(a, \alpha(a, i))=\alpha(a, i)$, so $i \sim k$. Moreover, since $i \sim k, \alpha(c, k)=\alpha(c, i)$, and we have that $\alpha(a, i)=\alpha(c, i)$. The converse is trivial, and the claim follows.

Let $K$ be a field. The commutative $K$-algebra $\bar{R}$ associated to $S$, constructed in $[\mathbf{1}]$, looks in this case as follows. Let $X=\left\{x_{i} \mid i \in I\right\}$, $Z=\left\{z_{a} \mid a \in A\right\}, Z^{\prime}=\left\{z_{a}^{\prime} \mid a \in A\right\}, Y=\{y\}, T=\{t\}, T^{\prime}=\left\{t^{\prime}\right\}$ and $R=K\left[X, Y, Z, T, Z^{\prime}, T^{\prime}\right]$. Let $M$ be the ideal of $R$ generated by:

- $t\left(x_{i}-x_{k}\right)$ for all $i, k \in I$ such that $i \sim k$,

- $t\left(z_{a} x_{i}-z_{c} x_{k}\right)$ for all $i, k \in I$ and all $a, c \in A$ such that $\alpha(a, i)=$ $\alpha(c, k)$,

- $y\left(z_{a}^{\prime}-z_{c}^{\prime}\right)$ for all $a, c \in A$.

Then $\bar{R}=R / M$.

Let $M^{\prime}$ be the ideal of $R$ generated by

- $t\left(x_{i}-x_{k}\right)$ for all $i, k \in I$ such that $i \sim k$,

- $t x_{i}\left(z_{a}-z_{c}\right)$ for all $i \in I$ and for all $a, c \in A$ such that $\alpha(a, i)=$ $\alpha(c, i)$,

- $y\left(z_{a}^{\prime}-z_{c}^{\prime}\right)$ for all $a, c \in A$. 
It is clear that $M^{\prime} \subseteq M$. Let $a, c \in A$ and $i, k \in I$ be such that $\alpha(a, i)=\alpha(c, k)$. Then,

$$
t\left(x_{i} z_{a}-x_{k} z_{c}\right)=t\left(x_{i}-x_{k}\right) z_{c}+t x_{i}\left(z_{a}-z_{c}\right) \in M^{\prime}
$$

since $i \sim k$ and $\alpha(a, i)=\alpha(c, i)$. Therefore $M=M^{\prime}$.

It is easy to check that $\alpha\left(a,\left(i_{1}, i_{2}\right)\right)=\alpha\left(c,\left(i_{1}, i_{2}\right)\right)$ if and only if $a=c$ or $\{a, c\}=\left\{i_{2}, i_{2}+1\right\}$. Moreover, it is clear that $\left(i_{1}, i_{2}\right) \sim\left(k_{1}, k_{2}\right)$ if and only if $i_{2}=k_{2}$. Therefore, $M$ is the ideal of $R$ generated by:

- $t\left(x_{\left(i_{1}, i_{2}\right)}-x_{\left(k_{1}, i_{2}\right)}\right)$ for all $i_{1}, k_{1}, i_{2} \in \mathbb{N}$,

- $t x_{\left(i_{1}, i_{2}\right)}\left(z_{i_{2}}-z_{i_{2}+1}\right)$ for all $i_{1}, i_{2} \in \mathbb{N}$,

- $y\left(z_{a}^{\prime}-z_{c}^{\prime}\right)$ for all $a, c \in A$.

Let $M^{\prime \prime}$ be the ideal of $R$ generated by

- $t\left(x_{\left(i_{1}, i_{2}\right)}-x_{\left(0, i_{2}\right)}\right)$ for all $i_{1}, i_{2} \in \mathbb{N}$,

- $t x_{\left(0, i_{2}\right)}\left(z_{i_{2}}-z_{i_{2}+1}\right)$ for all $i_{2} \in \mathbb{N}$,

- $y\left(z_{a}^{\prime}-z_{c}^{\prime}\right)$ for all $a, c \in A$.

It is clear that $M^{\prime \prime} \subseteq M$. Moreover,

$$
t\left(x_{\left(i_{1}, i_{2}\right)}-x_{\left(k_{1}, i_{2}\right)}\right)=t\left(x_{\left(i_{1}, i_{2}\right)}-x_{\left(0, i_{2}\right)}\right)-t\left(x_{\left(k_{1}, i_{2}\right)}-x_{\left(0, i_{2}\right)}\right) \in M^{\prime \prime}
$$

and

$$
\begin{array}{r}
t x_{\left(i_{1}, i_{2}\right)}\left(z_{i_{2}}-z_{i_{2}+1}\right)=t\left(x_{\left(i_{1}, i_{2}\right)}-x_{\left(0, i_{2}\right)}\right) z_{i_{2}}-t\left(x_{\left(i_{1}, i_{2}\right)}-x_{\left(0, i_{2}\right)}\right) z_{i_{2}+1} \\
+t x_{\left(0, i_{2}\right)}\left(z_{i_{2}}-z_{i_{2}+1}\right) \in M^{\prime \prime}
\end{array}
$$

Therefore, $M=M^{\prime \prime}$.

We will see that $S$ is a linear band and that there exists an infinite chain of annihilators in $\bar{R}=R / M$. We denote by $\bar{r}$ the image of $r$ under the natural map $R \rightarrow R / M$. Let $J_{k}=\operatorname{ann}_{\bar{R}}\left(\overline{t x_{(0,0)} x_{(0,1)} \cdots x_{(0, k)}}\right)$ for all $k \in \mathbb{N}$.

Clearly $J_{0} \subseteq J_{1} \subseteq \cdots$ and $\left(\overline{z_{k}-z_{k+1}}\right) \in J_{k}\left(\right.$ since $t x_{(0, k)}\left(z_{k}-z_{k+1}\right) \in$ $M)$. Let us check now that $\overline{\left(z_{k}-z_{k+1}\right)} \notin J_{k-1}$ for all $k \geq 1$.

Suppose that $\overline{\left(z_{k}-z_{k+1}\right)} \in J_{k-1}$ for some $k \geq 1$, that is,

$$
\gamma=\left(z_{k}-z_{k+1}\right) t x_{(0,0)} \cdots x_{(0, k-1)} \in M
$$

Then, there exist $p_{1}, p_{2}, q_{1}, q_{2}, q_{3} \in \mathbb{N}, \mu_{r}, \mu_{l, n, m}, \mu_{s}^{\prime} \in K \backslash\{0\}$, monomials $w_{s}^{\prime}, w_{r}, w_{l, n, m} \in R$ and $i_{r}, j_{m}, l_{n} \in \mathbb{N}, k_{1, s}, k_{2, s} \in \mathbb{N} \cup\{\infty\}$ for all $1 \leq r \leq$ 
$p_{1}, 1 \leq s \leq p_{2}, 1 \leq l \leq q_{1}, 1 \leq n \leq q_{2}, 1 \leq m \leq q_{3}$, such that

$$
\begin{aligned}
\gamma= & \sum_{r=1}^{p_{1}} \mu_{r} w_{r} t x_{\left(0, i_{r}\right)}\left(z_{i_{r}}-z_{i_{r}+1}\right) \\
& +\sum_{l=1}^{q_{1}} \sum_{n=1}^{q_{2}} \sum_{m=1}^{q_{3}} \mu_{l, n, m} w_{l, n, m} t\left(x_{\left(j_{m}, l_{n}\right)}-x_{\left(0, l_{n}\right)}\right) \\
& +\sum_{s=1}^{p_{2}} \mu_{s}^{\prime} w_{s}^{\prime} y\left(z_{k_{1, s}}^{\prime}-z_{k_{2, s}}^{\prime}\right) .
\end{aligned}
$$

Let $\psi: R \rightarrow R$ be the morphism of $K$-algebras such that $\psi\left(x_{\left(s_{1}, s_{2}\right)}\right)=$ $x_{\left(0, s_{2}\right)}$ for all $s_{1}, s_{2} \in \mathbb{N}, \psi\left(z_{a}^{\prime}\right)=z_{\infty}^{\prime}$ for all $a \in A$ and $\psi(w)=w$ for all $w \in Y \cup Z \cup T \cup T^{\prime}$. Then,

$$
\psi(\gamma)=\gamma=\psi\left(\sum_{r=1}^{p_{1}} \mu_{r} w_{r} t x_{\left(0, i_{r}\right)}\left(z_{i_{r}}-z_{i_{r}+1}\right)\right) .
$$

Therefore, we may assume that

$$
\gamma=\sum_{r=1}^{p_{1}} \mu_{r} w_{r} t x_{\left(0, i_{r}\right)}\left(z_{i_{r}}-z_{i_{r}+1}\right) .
$$

Let $U=\left\{1 \leq r \leq p_{1} \mid t w_{r} x_{\left(0, i_{r}\right)}=t x_{(0,0)} \cdot \ldots \cdot x_{(0, k-1)}\right\}$. It is clear that

$$
\gamma=\sum_{r \in U} \mu_{r} w_{r} t x_{\left(1, i_{r}\right)}\left(z_{i_{r}}-z_{i_{r}+1}\right)
$$

Note that, for $r \in U, 1 \leq i_{r} \leq k-1$. Therefore, the degree in $z_{k+1}$ of

$$
\sum_{r \in U} \mu_{r} w_{r} t x_{\left(1, i_{r}\right)}\left(z_{i_{r}}-z_{i_{r}+1}\right)
$$

is zero, which is a contradiction since

$$
\gamma=\left(z_{k}-z_{k+1}\right) t x_{(1,1)} \cdots x_{(1, k-1)} \text {. }
$$

Thus, $\overline{\left(z_{k}-z_{k+1}\right)} \notin J_{k-1}$ and $J_{0} \subsetneq J_{1} \subsetneq J_{2} \subsetneq J_{3} \subsetneq \cdots$ is an infinite chain of annihilators in $\bar{R}$. Then, $\bar{R}$ cannot be embedded into any Noetherian ring.

Finally, we shall see that $S$ is linear, that is, there exist a field $Q$, a positive integer $n$ and a monomorphism $\varphi: S \hookrightarrow M_{n}(Q)$.

Let $L$ be an arbitrary field and $D=L\left[\left\{x_{m} \mid m \in \mathbb{N}\right\}\right]$. Since $D$ is an integral domain, there exists its field of quotients $Q$. Now, we can define 
a function $\varphi: S \rightarrow M_{4}(Q)$ by

$$
\varphi\left(e_{\infty}\right)=\left(\begin{array}{cccc}
0 & 0 & 0 & 0 \\
0 & 1 & 0 & 0 \\
0 & 0 & 1 & 0 \\
0 & 0 & 0 & 1
\end{array}\right), \quad \varphi\left(e_{a}\right)=\left(\begin{array}{cccc}
0 & x_{a+1} & x_{a} & 0 \\
0 & 1 & 0 & 0 \\
0 & 0 & 1 & 0 \\
0 & 0 & 0 & 1
\end{array}\right)
$$

for all $a \in \mathbb{N}$,

$$
\varphi\left(f_{(m+1, m)}\right)=\left(\begin{array}{cccc}
0 & 0 & 0 & 0 \\
0 & 0 & 0 & x_{m}-x_{m+1} \\
0 & 0 & 0 & x_{m+2}-x_{m+1} \\
0 & 0 & 0 & 1
\end{array}\right)
$$

for all $m \in \mathbb{N}$ and

$$
\varphi\left(f_{(n, m)}\right)=\left(\begin{array}{cccc}
0 & 0 & 0 & x_{n+1}\left(x_{m}-x_{m+1}\right)+x_{n}\left(x_{m+2}-x_{m+1}\right) \\
0 & 0 & 0 & x_{m}-x_{m+1} \\
0 & 0 & 0 & x_{m+2}-x_{m+1} \\
0 & 0 & 0 & 1
\end{array}\right)
$$

for all $n, m \in \mathbb{N}$ with $n \neq m+1$.

We need to see that $\varphi$ is a monomorphism. First we shall see that $\varphi$ is injective. Clearly, if $\varphi(s)=\varphi\left(s^{\prime}\right)$, we have that $s$ and $s^{\prime}$ are in the same component. Let $e_{a}, e_{c} \in E, f_{\left(i_{1}, i_{2}\right)}, f_{\left(k_{1}, k_{2}\right)} \in F$ with $i_{1} \neq i_{2}+1$ and $k_{1} \neq k_{2}+1$.

If $\varphi\left(e_{a}\right)=\varphi\left(e_{c}\right)$, then, clearly, $e_{a}=e_{c}$.

If $\varphi\left(f_{\left(i_{1}, i_{2}\right)}\right)=\varphi\left(f_{\left(k_{1}, k_{2}\right)}\right)$, then $k_{2}=i_{2}$ and

$$
\begin{aligned}
x_{i_{1}+1}\left(x_{i_{2}}-x_{i_{2}+1}\right)+x_{i_{1}} & \left(x_{i_{2}+2}-x_{i_{2}+1}\right) \\
& =x_{k_{1}+1}\left(x_{i_{2}}-x_{i_{2}+1}\right)+x_{k_{1}}\left(x_{i_{2}+2}-x_{i_{2}+1}\right) .
\end{aligned}
$$

Since $i_{1} \neq i_{2}+1$, we have that

$$
x_{i_{1}+1} x_{i_{2}} \in\left\{x_{k_{1}+1} x_{i_{2}}, x_{k_{1}+1} x_{i_{2}+1}, x_{k_{1}} x_{i_{2}+1}\right\} .
$$

Therefore $i_{1} \in\left\{k_{1}, i_{2}\right\}$. Similarly, we can see that $k_{1} \in\left\{i_{1}, i_{2}\right\}$. Hence $i_{1}=k_{1}$ and $f_{\left(i_{1}, i_{2}\right)}=f_{\left(k_{1}, k_{2}\right)}$.

If $\varphi\left(f_{\left(i_{2}+1, i_{2}\right)}\right)=\varphi\left(f_{\left(k_{2}+1, k_{2}\right)}\right)$, then $k_{2}=i_{2}$ so $f_{\left(i_{2}+1, i_{2}\right)}=f_{\left(k_{2}+1, k_{2}\right)}$.

Therefore, $\varphi$ is injective.

Finally, we shall see that $\varphi\left(s_{1} s_{2}\right)=\varphi\left(s_{1}\right) \varphi\left(s_{2}\right)$ for all $s_{1}, s_{2} \in S$. If $e, e^{\prime} \in E, f \in F$ and $s \in S$, it is clear that $\varphi\left(e e^{\prime}\right)=\varphi(e) \varphi\left(e^{\prime}\right)$ and $\varphi(f s)=\varphi(f) \varphi(s)$. Let $a, n, m \in \mathbb{N}$. 


$$
\begin{aligned}
& \text { If } a \neq m+1 \text {, then } e_{a} f_{(n, m)}=f_{(a, m)} \text { and } \\
& \begin{aligned}
\varphi\left(e_{a} f_{(n, m)}\right) & =\left(\begin{array}{cccc}
0 & 0 & 0 & x_{a+1}\left(x_{m}-x_{m+1}\right)+x_{a}\left(x_{m+2}-x_{m+1}\right) \\
0 & 0 & 0 & x_{m}-x_{m+1} \\
0 & 0 & 0 & x_{m+2}-x_{m+1} \\
0 & 0 & 0 & 1
\end{array}\right) \\
& =\varphi\left(e_{a}\right) \varphi\left(f_{(n, m)}\right) .
\end{aligned}
\end{aligned}
$$

Since $e_{m+1} f_{(n, m)}=f_{(m, m)}$, we have

$$
\varphi\left(e_{m+1} f_{(n, m)}\right)=\left(\begin{array}{cccc}
0 & 0 & 0 & x_{m} x_{m+2}-x_{m+1}^{2} \\
0 & 0 & 0 & x_{m}-x_{m+1} \\
0 & 0 & 0 & x_{m+2}-x_{m+1} \\
0 & 0 & 0 & 1
\end{array}\right)=\varphi\left(e_{m+1}\right) \varphi\left(f_{(n, m)}\right),
$$

and, since $e_{\infty} f_{(n, m)}=f_{(m+1, m)}$,

$$
\varphi\left(e_{\infty} f_{(n, m)}\right)=\left(\begin{array}{cccc}
0 & 0 & 0 & 0 \\
0 & 0 & 0 & x_{m}-x_{m+1} \\
0 & 0 & 0 & x_{m+2}-x_{m+1} \\
0 & 0 & 0 & 1
\end{array}\right)=\varphi\left(e_{\infty}\right) \varphi\left(f_{(n, m)}\right) .
$$

Therefore, $\varphi$ is an injective homomorphism and $S$ is a linear band.

\section{References}

[1] F. CEDó AND J. OKNIŃski, Faithful linear representations of bands, Publ. Mat. 53(1) (2009), 119-140.

[2] F. Cedó And E. Rodríguez-Jorge, Rings associated to bands with two components, Comm. Algebra (to appear).

[3] K. Cvetкo-Vah, Skew lattices of matrices in rings, Algebra Universalis 53(4) (2005), 471-479.

[4] L. Livshits, G. MacDonald, B. Mathes, and H. Radjavi, Reducible semigroups of idempotent operators, J. Operator Theory 40(1) (1998), 35-69.

[5] J. OkNiŃski, "Semigroups of matrices", Series in Algebra 6, World Scientific Publishing Co., Inc., River Edge, NJ, 1998.

[6] M. Petrich, "Lectures in semigroups", Mathematische Lehrbücher und Monographien. I. Abteilung: Mathematische Lehrbücher 29, Akademie-Verlag, Berlin, 1977.

[7] H. Radjavi And P. Rosenthal, "Simultaneous triangularization", Universitext, Springer-Verlag, New York, 2000. 
Departament de Matemàtiques

Universitat Autònoma de Barcelona

08193 Bellaterra (Barcelona)

Spain

E-mail address: cedo@mat.uab.cat

E-mail address: erguez@mat.uab.cat

Primera versió rebuda el 5 de novembre de 2009, darrera versió rebuda el 17 de febrer de 2010. 